\title{
Analyses of SELEX-derived ZAP-binding RNA aptamers suggest that the binding specificity is determined by both structure and sequence of the RNA
}

\author{
Zhi Huang, Xinlu Wang, Guangxia Gao
}

Key Laboratory of Infection and Immunity, Institute of Biophysics, Chinese Academy of Sciences, Beijing 100101, China $\triangle$ Correspondence: gaogx@moon.ibp.ac.cn

Received June 10, 2010 Accepted July 14, 2010

\section{ABSTRACT}

The zinc-finger antiviral protein (ZAP) is a host factor that specifically inhibits the replication of certain viruses, including murine leukemia virus, Sindbis virus and Ebola virus, by targeting the viral mRNAs for degradation. ZAP directly binds to the target viral mRNA and recruits the cellular RNA degradation machinery to degrade the RNA. No significant sequence similarity or obvious common motifs have been found in the so far identified target viral mRNAs. The minimum length of the target sequence is about $500 \mathrm{nt}$ long. Short workable ZAP-binding RNAs should facilitate further studies on the ZAP-RNA interaction and characterization of such RNAs may provide some insights into the underlying mechanism. In this study, we used the SELEX method to isolate ZAP-binding RNA aptamers. After 21 rounds of selection, ZAP-binding aptamers were isolated. Sequence analysis revealed that they are G-rich RNAs with predicted stem-loop structures containing conserved "GGGUGG" and "GAGGG" motifs in the loop region. Insertion of the aptamer sequence into a luciferase reporter failed to render the reporter sensitive to ZAP. However, overexpression of the aptamers modestly but significantly reduced ZAP's antiviral activity. Substitution of the conserved motifs of the aptamers significantly impaired their ZAP-binding ability and ZAP-antagonizing activity, suggesting that the RNA sequence is important for specific interaction between ZAP and the target RNA. The aptamers identified in this report should provide useful tools to further investigate the details of the interaction between ZAP and the target RNAs. KEYWORDS zinc-finger antiviral protein (ZAP),
SELEX, RNA aptamers

\section{INTRODUCTION}

The zinc-finger antiviral protein (ZAP) was initially recovered as a host factor that inhibited the infection of cells by Moloney murine leukemia virus (MLV) (Gao et al., 2002). In addition to MLV, ZAP also inhibits the replication of Ebola virus (EBOV) and Marburg virus (MARV) (Muller et al., 2007), and several members of the Alphavirus genus, including Sindbis virus (SINV) (Bick et al., 2003). However, ZAP does not induce a universal antiviral state, as the replications of some viruses, including herpes simplex virus type 1 and yellow fever virus, are not affected in ZAP-expressing cells (Bick et al., 2003).

ZAP directly binds to specific viral mRNA sequences (Guo et al., 2004) and recruits the RNA processing exosome to degrade the target RNA (Guo et al., 2007). The RNA helicase p72 is required for optimal function of ZAP (Chen et al., 2008). Insertion of some viral sequences into the 3' UTR of a luciferase reporter rendered the reporter sensitive to ZAP's inhibitory effect (Guo et al., 2004). The ZAP responsive element (ZRE) in MLV was mapped to the $3^{\prime}$ long terminal repeat, and the ZREs in SINV were mapped to multiple fragments (Guo et al., 2004). The sensitive sequences in EBOV and MARV were mapped to the $L$ fragment (Muller et al., 2007). The only common feature of these ZAP target sequences is that they are all more than $500 \mathrm{nt}$ long. No obvious common elements or motifs can be identified in these ZREs. A short workable ZAP-binding RNA is needed for further studies on the interaction between ZAP and the target RNA. 
The Systematic Evolution of Ligands by Exponential enrichment (SELEX) is an efficient method to isolate highaffinity DNA or RNA ligands for target proteins (Jayasena, 1999). RNA aptamers isolated by SELEX can adopt complex structures to bind target proteins with high affinities (Ellington and Szostak, 1990; Tuerk and Gold, 1990). For example, using the SELEX method RNA aptamers have been isolated that bind HIV Tat (Yamamoto et al., 2000) and reverse transcriptase (Tuerk et al., 1992) and these aptamers proved to be powerful tools for better understanding the RNA-protein interactions.

In this report, as a step towards understanding the interaction between ZAP and its target RNA, the SELEX method was used to isolate RNA aptamers that bind to ZAP and these aptamers were further characterized.

\section{RESULTS}

\section{Isolation of ZAP-binding RNA aptamers by SELEX}

To generate an RNA library for SELEX, oligonucleotides containing 40 nucleotides of random sequence flanked by defined sequences were PCR-amplified, with the T7 promoter sequence built in the forward primer (Fig. 1A). The amplified DNA was transcribed to produce a random RNA library. The RNA library was incubated with myc-tagged ZAP immobilized on Protein G-agarose via anti-myc antibody. The bound RNAs were recovered, reverse transcribed and PCR-amplified. The PCR product was used as template to generate RNAs for the next round of selection. A total of 21 rounds of selection were performed.

To confirm that the selected RNAs had higher binding affinity for ZAP, the unselected RNA (pool 0) and selected RNA (pool 21) were assayed for their binding to ZAP. The pool 21 RNA bound to ZAP could be easily detected and the amount of the bound RNA increased as the amount of ZAP increased, while little pool 0 RNA bound to ZAP although comparable amounts of ZAP protein were used as determined by Western blotting (Fig. 1B). In the absence of ZAP only very weak background binding was detected (Fig. 1C). These results indicated that ZAP-binding RNA aptamers were successfully selected.

\section{Sequence analysis of the selected RNA aptamers}

Twenty nine clones randomly picked from round 21 were sequenced. All of them are rich in guanidine and all except two contain "GGGUGG" and/or "GAGGG" motifs. Based on sequence alignment, the RNA aptamers were divided into three groups (Fig. 2A). The aptamers containing the motif "GAGGGUGGG" were grouped as group I, the aptamers containing the motif "GGGGUGGAGGG" were grouped as group II, and the rest aptamers were grouped as group III. The MFold program (Zuker, 2003) was used to predict the minimum-energy structures of clones $21 \mathrm{E}, 21 \mathrm{~K}$ and $21 \mathrm{I}$, which were chosen as representatives of groups I, II and III, respectively. Since the RNAs subjected to selection contained the vector sequence, the sequences from both the vector and library were used for structure prediction. All the three aptamers were predicted to form stem-loop like structures. The sequences of the loop region were derived from the library and the sequences of the stem region were derived from the vector and library. Since the vector sequence was fixed, it seems that pairing sequences were selected from the library to form the stem. In the loop region, there are two highly conserved motifs, "GGGUGG" and "GAGGG" (Fig. 2B). The aptamers in groups I and II were more abundant than those in group III. Hence, aptamers 21E and $21 \mathrm{~K}$ were chosen for further analyses.

\section{Overexpression of the ZAP-binding RNA aptamers reduced ZAP's antiviral activity}

The viral ZREs can confer to the pGL3-luc reporter sensitivity to ZAP's inhibitory effect (Guo et al., 2004). To test whether the RNA aptamers had such a function, the sequence encoding $21 \mathrm{E}$ or $21 \mathrm{~K}$ was cloned into the reporter. The reporter carrying a ZAP-sensitive fragment from SINV (SINV-M) was used as a positive control, and the reporter carrying a random fragment from pool 0 was used as a negative control. The sensitivity of the reporter was indicated by fold inhibition. While the positive control SINV-M conferred to the reporter significant sensitivity to ZAP, $21 \mathrm{E}$ or $21 \mathrm{~K}$ failed to do so (Fig. 3A). A reporter containing two copies of $21 \mathrm{E}$ was also tested and it did not display any detectable increased sensitivity to ZAP (Fig. 3A). This result indicated that the ZAP-binding aptamers were not sufficient to confer sensitivity to the reporter.

Considering the binding ability of the aptamers to ZAP, we reasoned that overexpression of the aptamers in ZAPexpressing cells might competitively inhibit the binding of ZAP to the target viral mRNA and thereby inhibit the antiviral activity of ZAP. The aptamer 21E was first analyzed for its ability to inhibit the binding of a viral ZRE to ZAP in the in vitro binding assay. Indeed, 21E RNA inhibited the binding of the viral ZRE to ZAP in a dose dependent manner (Fig. 3B). To test the inhibitory activity of the aptamer in vivo, the sequence encoding aptamer 21E was cloned into pSuper-Retro. The RNA aptamer-expressing plasmid was cotransfected into 293TRex-ZAP cells with the pMLV-luc reporter and assayed for the effect on ZAP's activity. Compared with the random RNA, overexpression of the aptamer 21E modestly but significantly reduced ZAP's activity (Fig. 3C). The same experiment was also performed in Rat2Trex-ZAP cells, in which ZAP was expressed in a tetracycline-inducible manner in Rat2 cells, and similar result was observed (Fig. 3C). These results indicated that overexpression of the ZAP-binding aptamer inhibited ZAP's activity. 
A forward primer

$5^{\prime}$-TAATACGACTCACTATAGGGAACAGTCCGAGCC-3'

$5^{\prime}$-GGGAACAGTCCGAGCC---N40---GGGTGAATTCGTCATA-3'

$3^{\prime}$-CCCACTTAAGCAGTAT-5'

PCR

reverse primer

T7 promoter

$5^{\prime}$-TAATACGACTCACTATA GGGAACAGTCCGAGCC---N40---GGGTGAATTCGTCATA-3'

3' ATTATGCTGAGTGATAT CCCTTGTCAGGCTCGG---N40---CCCACTTAAGCAGTAT-5'

in vitro transcription

GGGAACAGUCCGAGCC---N40--GGGUGAAUUCGUCAUA-3' RNA library

B

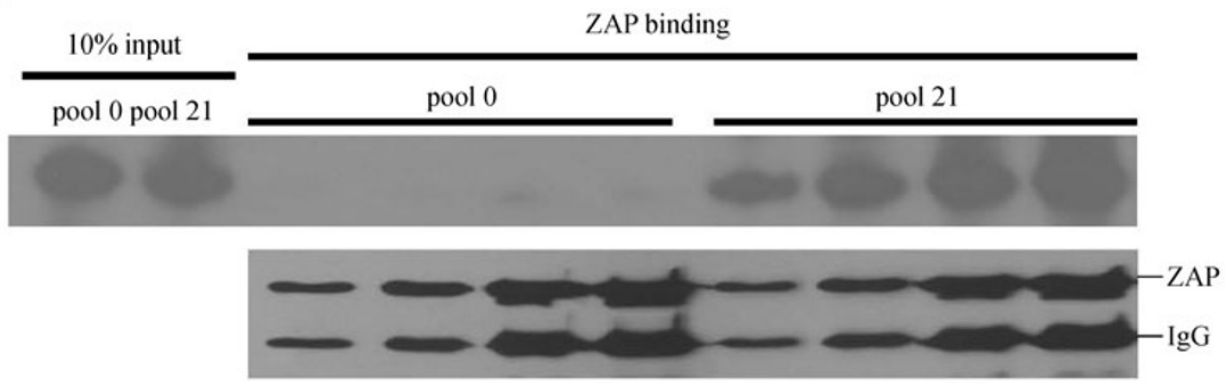

C

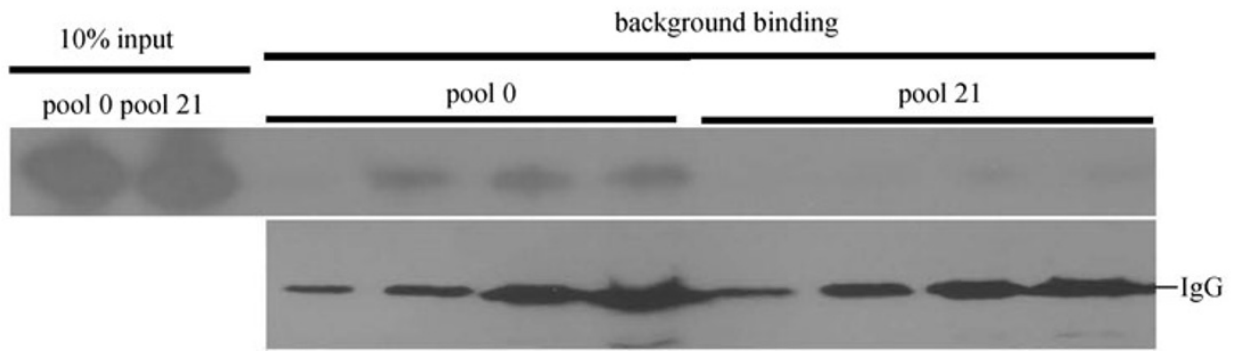

Figure 1. In vitro selection of ZAP-binding RNA aptamers. (A) Strategy for producing the RNA library for selection of ZAPbinding RNA aptamers. The synthetic oligonucleotides containing 40 nucleotides of random sequence (N40) were PCR-amplified. The PCR product was used as template to generate the RNA library by in vitro transcription. (B and C) Confirmation that the selected RNA had higher ZAP-binding affinity. The initial library RNA (pool 0) and the RNA after 21 rounds of selection (pool 21) were labeled with [ $\alpha-32 P] U T P$ by in vitro transcription, and incubated with increasing amounts of the lysates of myc-tagged ZAP-expressing cells (B) or control cells (C) immobilized on agarose resin. The resins were washed, resuspended, and divided into two fractions, $80 \%$ for detection of the bound RNA by urea-PAGE followed by exposure to X-ray film (upper panel), and the rest $20 \%$ for detection of ZAP by Western blotting (lower panel).

The "GAGGG" and "GGGUGG" motifs were required for the RNA aptamers to bind ZAP

In the aptamers of group I and II, there are two conserved motifs, GAGGG and GGGUGG. To analyze their function, they were mutated in aptamers $21 \mathrm{~K}$ and 21E. The GAGGGUGG motif in 21E was replaced with AAAAAAAA, and the GGGUGG and GAGGG motifs in $21 \mathrm{~K}$ were replaced with AAAAAA and AAAAA, respectively. These substitutions were predicted not to change the secondary structures (data not 
A

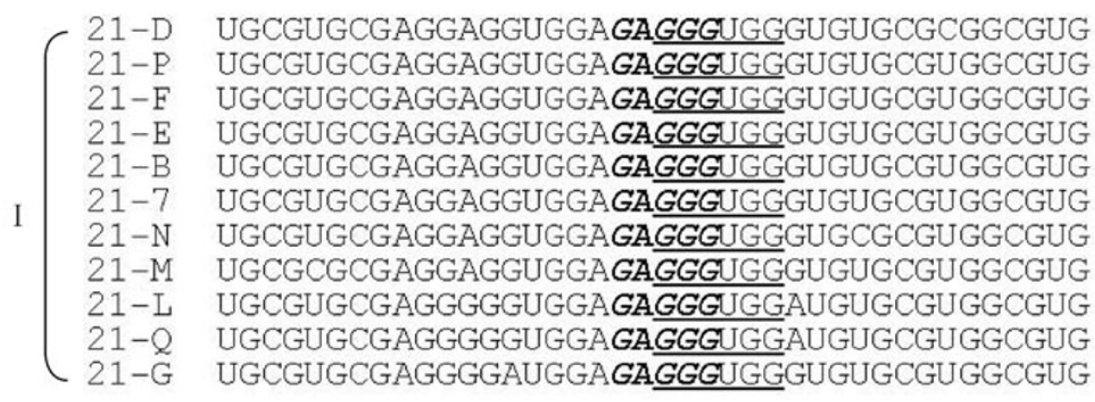

II $\left(\begin{array}{lll}21-9 & \text { AAGCGGUAGCGUCAGGGGGUGGAGGGAGGAGGCCGCGUGGUGU } \\ 21-\mathrm{K} & \text { AAGCGGUAGCGUCAGGGGGUGGAGGGAGGAGGCCGCGUGGUGU } \\ 21-0 & \text { AAGCGGUAGCGUCAGGGGGUGAGGGAGGAGGCCGCGUGGUGU } \\ 21-3 & \text { AAGCGGUAGCGUCAGGGGGUGGAGGGAAGGAGGCCGCGUGGUGU } \\ 21-\mathrm{A} & \text { AAGCGGUAGCGUCAGGGGUGGAGGGAGGAGGCCGCGUGGUGG } \\ 21-\mathrm{T} & \text { AAGCGGUAGCGUCAGGGGGUGGAGGGAGGAGGCCGCGUGGUGG }\end{array}\right.$

III $\left(\begin{array}{lll}21-\text { I } & \text { GCGGGGAGGGUUAGGGUGGUGUGGAGCGAGCGGUGGGCGUG } \\ 21-4 & \text { AGGAGGGGGAUUGCGCGAGGGUAAGGGUGGCGUGUCGGUGU } \\ 21-\mathrm{H} & \text { AGGAGGGGGAUUGCGCGAGGGUAAGGGUGGCGUGUCGGUGU } \\ 21-2 & \text { GUAGGAGAGGGUAGGGUAGGGUUGGCGCGUGGUGU } \\ 21-\mathrm{C} & \text { GUAGGAGAGGGUAGGGUAGGGUUGGCGCGUGGUGU } \\ 21-J & \text { CAUGGGUGGAAGAGGGUUGGGAGGGGUGCGGCUGUGGUGG } \\ 21-6 & \text { ACGGGGAGGAGGAGGGGGUAGUGGUUGGUGGGGCGGGGGGUG } \\ 21-5 & \text { AGGGGGGGGUUGGGAGGUACGGAUGGUAGCGGGCUGGGU } \\ 21-\mathrm{R} & \text { AGGGGUGGGUUGGGAGGUACGGAUGGUAGCGGGCUGGUGG } \\ 21-1 & \text { GCAGGGUAGGGUAGGGCAGGGUAUGGUUGGGACGUGGUGG } \\ 21-10 & \text { GCGGGAAAGGGUAGGGUGGGGUGGGGCGGGGGGGGGGGGGG } \\ 21-8 & \text { GAGGGGGGGAGAAGUGGAGGGUACGGUAUGCGGGCUGGGU }\end{array}\right.$

B

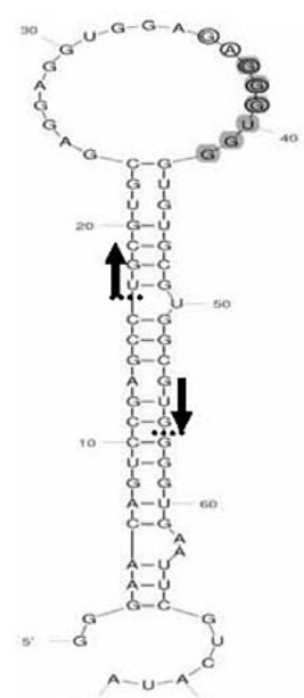

$21 \mathbf{E}^{\circ}: \mathrm{dG}=-18.40$

[initially -18.40 ]

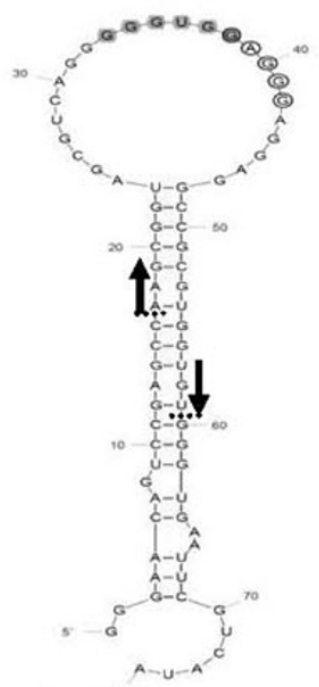

$21 \mathrm{~K}^{5} \mathrm{dG}=-17.50$

[initially -17.50 ]

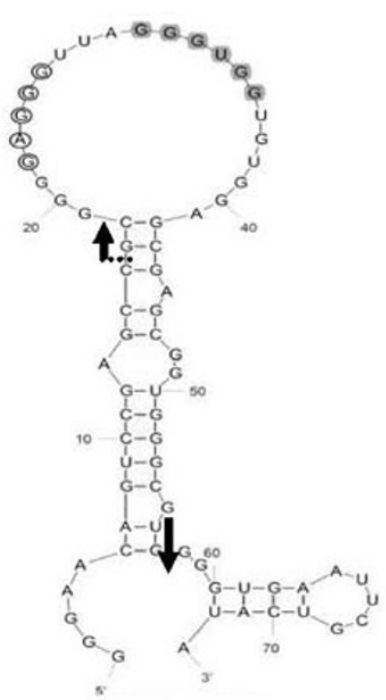

21I: $d G=-13.90$

[initially -14.40 ]

Figure 2. Sequence analysis of the selected RNA aptamers. (A) Multiple alignments of the selected RNA aptamers. The conserved motifs are in bold-type font or underlined. (B) Schematic representation of the secondary structures of aptamers $21 \mathrm{E}, 21 \mathrm{~K}$ and 21 l predicted by the MFold algorithm. The sequences derived from the library are indicated by the arrows. The conserved motifs are labeled with circles or shadows.

shown). The binding ability and inhibitory effects on ZAP of these aptamers were examined. All the mutant aptamers displayed significantly reduced binding to ZAP (Fig. 4A). Consistently, overexpression of these mutant aptamers had 

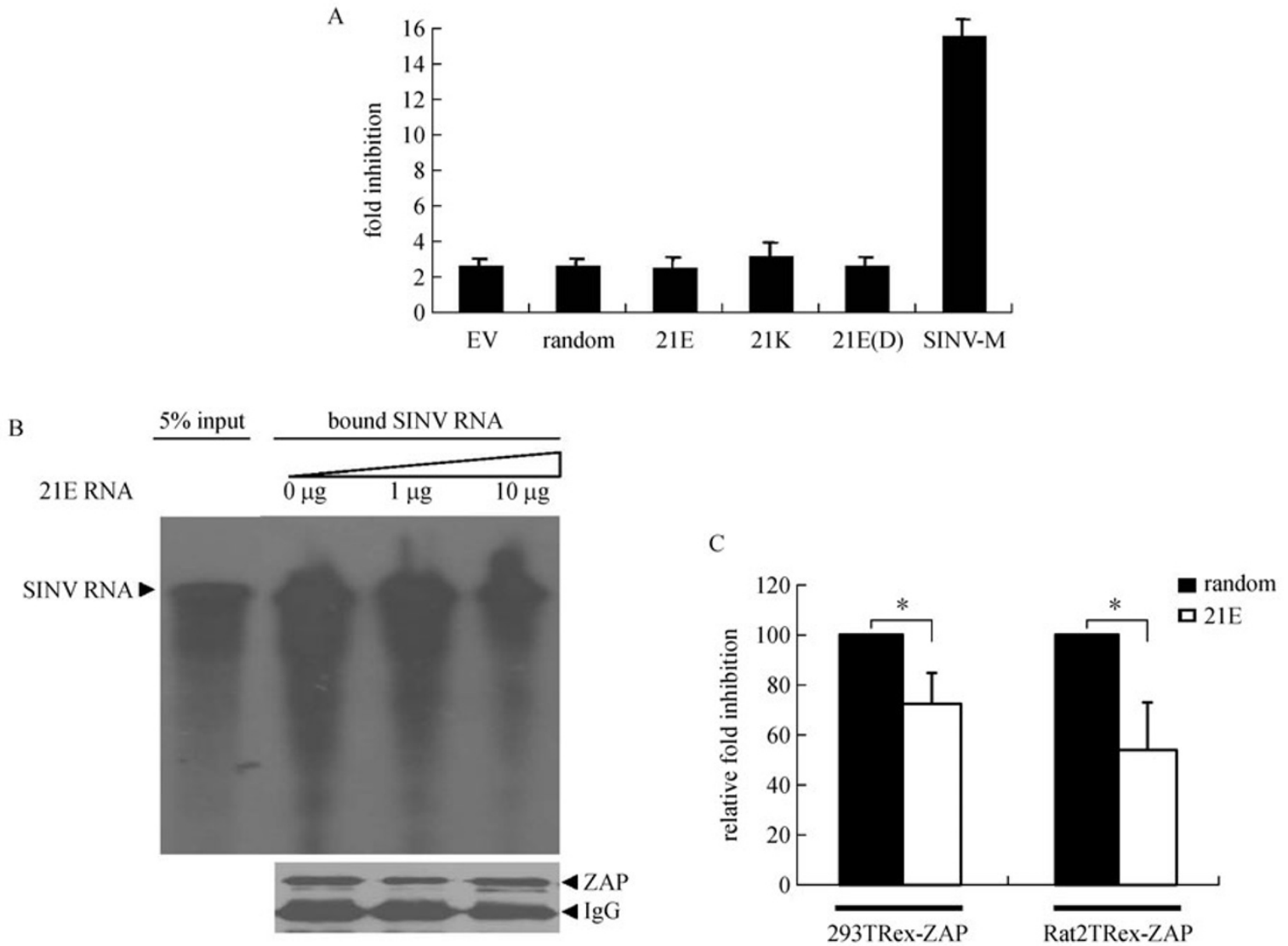

Figure 3. Functional assays for the ZAP-binding RNA aptamers. (A) The ZAP-binding RNA aptamer was not sufficient to confer to the reporter sensitivity to ZAP. The sequence encoding $21 \mathrm{E}, 21 \mathrm{~K}$ or a random RNA from unselected library was cloned into pGL3-Luc to generate reporters pGL3-21E, pGL3-21K and pGL3-random, respectively. The reporters were transfected into 293TRex-ZAP cells with pRL-TK, a Renilla luciferase reporter that is not affected by ZAP expression, to control transfection efficiency and sample handling. At $6 \mathrm{~h}$ posttransfection, the cells were equally divided into two dishes, with one mock treated and the other treated with tetracycline to induce ZAP expression. At $48 \mathrm{~h}$ postinfection, the cells were lysed and luciferase activities were measured. Fold inhibition was calculated as the normalized luciferase activity in the mock treated cells divided by the normalized luciferase activity in the tetracycline treated cells. pGL3-M: pGL3-luc-linker reporter containing the ZAP-sensitive fragment from SINV to serve as a positive control. (B) The RNA aptamer competitively inhibited the binding of ZAP to the target viral mRNA in vitro. The radio-labeled viral ZRE, $\mathrm{Na}$, was incubated with the lysate of myc-tagged ZAP-expressing cells immobilized on agarose resin. Increasing amounts of cold 21E RNA were also added to the binding buffer. The resins were washed, resuspended, and divided into two fractions, $80 \%$ for detection of the bound RNA by urea-PAGE followed by exposure to X-ray film (upper panel), and the rest $20 \%$ for detection of ZAP by Western blotting (lower panel). (C) Overexpression of the ZAP-binding RNA aptamers reduced ZAP's activity. The aptamer encoding sequence was cloned into $\mathrm{pSuper-Retro} \mathrm{to} \mathrm{generate} \mathrm{aptamer-expressing} \mathrm{plasmid} \mathrm{pSR-21E} \mathrm{or} \mathrm{pSR-}$ Random. The constructs were co-transfected into 293TRex-ZAP or Rat2TRex-ZAP cells with pMLV-luc and pRL-TK. At $6 \mathrm{~h}$ posttransfection, the cells were mock-treated or treated with tetracycline to induce ZAP expression. At $48 \mathrm{~h}$ posttransfection, luciferase activities were measured. Fold inhibition by ZAP was calculated as above described. The relative fold inhibition of ZAP in the presence of pSR-Random was arbitrarily set as $100 .{ }^{*}$ denotes $p<0.05, n=3$.

little effect on ZAP's activity (Fig. 4B). These results indicated that the conserved motifs were required for the aptamers to bind ZAP.

\section{DISCUSSION}

In this study, we used the SELEX method to isolate
ZAP-binding aptamers (Fig. 1A). Since a stable RNA secondary structure usually requires $30-40$ nucleotides, a library containing 40 random nucleotides was used. The selected ZAP-binding aptamers are all G-rich RNAs with predicted stem-loop structures containing conserved "GGGUGG" and "GAGGG" motifs in the loop region (Fig. 2). Although they lack any obvious similarity to the so far 
A

$10 \%$ input

ZAP binding

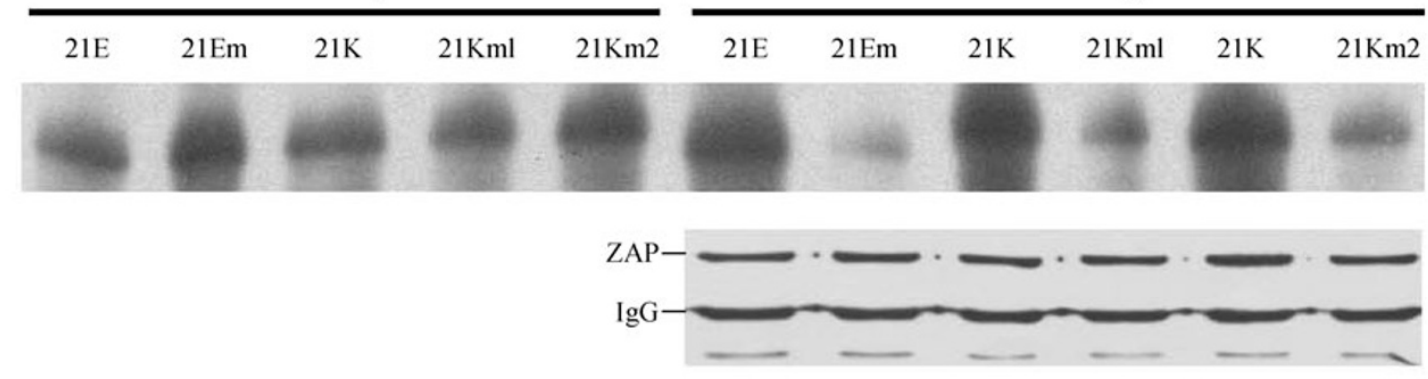

B

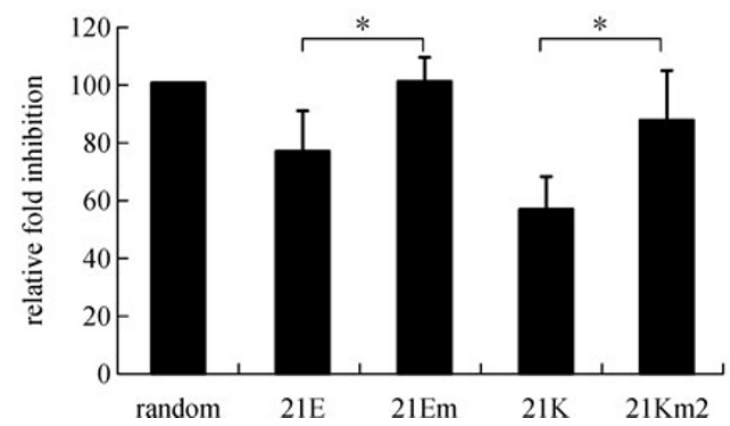

Figure 4. The GAGGG and GGGUGG motifs are required for the aptamers to bind to ZAP and thereby to inhibit ZAP's activity. (A) In vitro binding assay. The conserved motif GAGGGUGG in 21E was replaced with AAAAAAA to generate mutant 21Em. The conserved motifs GGGUGG and GAGGG were replaced with AAAAAA and AAAAA to generate $21 \mathrm{Km} 1 \mathrm{and} 21 \mathrm{Km} 2$, respectively. The indicated RNA aptamers were assayed for their binding to ZAP as described in the legend to Fig. 1B. (B) The aptamer coding sequence was cloned into pSuper-Retro and assayed for their ability to reduce ZAP's activity in 293TRex-ZAP cells as described in the legend to Fig. 3B. *denotes $p<0.05, n=3$.

identified viral ZREs (data not shown), analyses of the aptamers help to provide some insight into how ZAP interacts with the target RNA.

Insertion of the aptamer sequence into the pGI3-luc reporter failed to improve the reporter's sensitivity to ZAP (Fig. 3A). However, the aptamer competitively inhibited the binding of ZAP to a viral ZRE RNA in vitro and overexpression of the aptamer in ZAP-expressing cells modestly but significantly reduced ZAP's activity (Fig. 3B and 3C). These results suggested that the aptamer was able to bind ZAP both in vivo and in vitro, but not sufficient to confer to the reporter sensitivity to ZAP. The reporter containing two copies of $21 \mathrm{E}$ aptamer did not improve the sensitivity to ZAP, either (Fig. 3A). One possibility is that the binding affinity of the aptamer for ZAP was too low compared with the viral ZREs. It is conceivable that multiple sites of interaction between ZAP and RNA might be required for ZAP to tightly bind to the RNA and target it for degradation. This is consistent with the fact that all the ZREs so far identified are at least $500 \mathrm{nt}$ long, which are supposed to form tertiary structures and have multiple possible binding sites. The aptamer may specifically bind to limited sites of ZAP with low affinity.

In the loop region of the aptamers, there are two conserved motifs. Substitution of these motifs dramatically impaired the aptamer's ZAP-binding ability and ZAP-antagonizing activity (Fig. 4). The $\mathrm{CCCH}$-type zinc-finger motifs have been shown to be required for ZAP to bind the target RNA (Guo et al., 2004). Similar zinc-finger motifs also exist in tristetraproline (TTP) (DuBois et al., 1990), which specifically targets the AU-rich element (ARE) often found in the 3 ' untranslated regions of cytokines, growth factors and proto-oncogenes (Lai et al., 1999, 2000; Blackshear, 2002; Carrick et al., 2004; Barreau et al., 2005), and characterized by the AUUUA motif. These results suggest that sequence-specific interaction between ZAP and RNA is important for ZAP to bind the RNA. How ZAP interacts with specific RNA sequence awaits further investigation.

In conclusion, the SELEX-derived ZAP-binding aptamers were G-rich RNAs with predicted stem-loop structures containing conserved "GGGUGG" and "GAGGG" motifs in 
the loop region. Overexpression of the aptamers reduced ZAP's antiviral activity. The conserved motifs were required for aptamers' binding to ZAP, suggesting that the RNA sequence is important for specific binding to ZAP. The aptamers should provide useful tools for further investigating the details of the interaction between ZAP and the target RNAs.

\section{MATERIALS AND METHODS}

\section{Plasmid construction}

The luciferase reporter plasmid, pGI3-luc-linker, has been described previously (Guo et al., 2004). The plasmid pGI3-luc-M, which was generated by inserting a ZAP-sensitive fragment from SINV into the pGI3-luc-linker, has also been reported (Guo et al., 2004). pGl3-luc$21 \mathrm{E}$ is a reporter with the coding sequence of aptamer $21 \mathrm{E}$ inserted in the 3' UTR of luciferase. pGI3-luc-21E (D) contains two copies of the coding sequence of aptamer 21E. pGI3-luc-random is a reporter carrying a random sequence (5'-gggaacagtccgagccgcgggcgaggaaagctcgtatggtcgcacggctgtgtatggggtg-3') amplified from unselected pool 0. To generate pGI3-luc-21E and pGI3-luc-21E (D), the 21E aptamer cDNA was PCR amplified using primers $72 \mathrm{FP}$ and $72 \mathrm{RP}$ and cloned into pGL3-Luc-linker in the sense orientation using the BamHI site. The same strategy was employed to generate pGI3-luc-21K and pGI3-luc-random. The primer sequences are listed below:

72FP: 5'-TATAGGATCCGGGAACAGTCCGAGCC-3';

72RP: 5'-TATAGGATCCTATGACGAATTCACCCC-3'.

pSR-21E expresses aptamer 21E. To generate pSR-21E, the sequence encoding aptamer 21E was PCR-amplified using primers SR-FP and SR-RP and cloned into pSuper-Retro (Oligoengine) under the $\mathrm{H} 1$ promoter using the Bglll and Hindlll sites. The primer sequences are listed below:

SR-FP: 5' -GGAAGATCTGGGAACAGTCCGAGCC-3';

SR-RP: 5'-ATCCCAAGCTTAAAAATATGACGAATTCACCC-3'.

\section{Preparation of random RNA library}

The synthetic oligonucleotides containing 40 nucleotides of random sequence flanked by defined sequences were PCR-amplified, with the T7 promoter sequence built in the forward primer. The amplified DNA was purified by phenol and chloroform extraction followed by ethanol precipitation, and transcribed with T7 RNA polymerase (T7 RiboMAX. Express Large Scale RNA Production System, Promega). The sequences of the primers are shown in Fig. $1 \mathrm{~A}$.

\section{In vitro selection of RNA aptamers}

293TRex-ZAP cells were treated with $1 \mu \mathrm{g} / \mathrm{mL}$ tetracycline for $12 \mathrm{~h}$ to induce ZAP expression, and then lysed in lysis buffer A ( $25 \mathrm{mM}$ Trisphosphate, $\mathrm{pH}$ 7.8, $2 \mathrm{mM}$ dithiothreitol, $2 \mathrm{mM}$ 1,2-diaminocyclohexane- N,N,N',N'-tetraacetic acid, 10\% glycerol, 1\% Triton X-100). The lysates were clarified by centrifugation at $13,000 \mathrm{rpm}$ (Sorvall Biofuge, Fresco) for $10 \mathrm{~min}$. The myc-tagged ZAP was immobilized on protein G-agarose resin (Santa Cruz) using the 9E10 anti-myc antibody (Santa Cruz). The resin was washed three times in RNase- free binding buffer (10 mM Tris-HCl, pH 7.5, $50 \mathrm{mM} \mathrm{NaCl}, 1 \mathrm{mM}$ EDTA and $10 \mu \mathrm{M} \mathrm{ZnCl}_{2}$ ).

Approximately $20 \mathrm{nM}$ of the RNA library was first incubated with $100 \mu \mathrm{L}$ of protein G-agarose resin in the RNase-free binding buffer for $20 \mathrm{~min}$ at room temperature with occasional shaking. The resin was discarded and the precleared RNA was incubated with immunoimmobilized ZAP protein in $500 \mu \mathrm{L}$ of the binding buffer supplemented with RNasin (Promega), $1 \mu \mathrm{g} / \mu \mathrm{L}$ heparin and $200 \mathrm{ng} / \mu \mathrm{L}$ yeast tRNA for $30 \mathrm{~min}$ at room temperature. The resin was washed three times with the binding buffer, resuspended, and treated with $0.5 \%$ SDS and protease $\mathrm{K}$ at $55^{\circ} \mathrm{C}$ for 30 min to detach the bound RNA from the resin. The RNA was recovered by phenol-chloroform extraction followed by ethanol precipitation. The recovered RNAs were reverse transcribed with MLV reverse transcriptase (Invitrogen), and amplified by PCR with Taq DNA polymerase for the next round of selection.

After the 21st round of selection, the cDNAs were amplified by PCR, cloned into pMD18T vector (Takara) and sequenced. The ClustalW software was used for multiple alignments of these RNA aptamers. The secondary structures of the selected RNA aptamers were predicted by the MFold program based on the Zuker algorithm (Zuker, 2003).

\section{In vitro binding assay}

The aptamer RNA was labeled with [a-32P] UTP (Amersham) by in vitro transcription using the Riboprobe Systems following the manufacture's instruction (Promega). The labeled RNA was purified by Sephadex G25 spin columns (Roche Diagnostics). $1 \times 10^{5} \mathrm{cpm}$ labeled RNA was incubated with $30 \mu \mathrm{L}$ immuno-immobilized ZAP in the binding buffer as described above. The resin was washed three times with the binding buffer, resuspended, and divided into two fractions, $80 \%$ for detection of the bound RNA by urea-PAGE and $20 \%$ for detection of ZAP protein by Western blotting.

\section{Cell culture and sensitivity assay}

All the cells were maintained in DMEM supplemented with $10 \%$ FBS. Transfection was performed using Lipofectamine 2000 following the manufacturer's instruction (Invitrogen). 293TRex-ZAP cell line has been described previously (Guo et al., 2004). To establish the Rat2TRex-ZAP cell line, Rat2TRex cells, which stably express the repressor of the Tet operon, were stably transfected with pcDNA4/TO/ myc-ZAP (Guo et al., 2004) and selected for Zeocin resistance. Individual clones were picked, expanded, and tested for tetracyclineinducible expression of ZAP by Western blotting.

The luciferase reporter assay has been previously reported (Guo et al., 2004). Briefly, pGI3-luc-21E, pGl3-luc-21K or pGl3-luc-random was transfected into 293Trex-ZAP together with pRL-TK (Promega). At $6 \mathrm{~h}$ post transfection, the cells were equally divided into two dishes, with one mock treated and the other treated with tetracycline to induce ZAP expression. At $48 \mathrm{~h}$ post transfection, the cells were lysed and luciferase activities were measured. The expression level of $p R L-T K$, a plasmid expressing Renilla luciferase that is not affected by ZAP, was used to normalize the transfection efficiencies. Fold inhibition was calculated as the normalized luciferase activity in the mock treated cells divided by the normalized luciferase activity in the tetracycline treated cells.

To assay the effect of the expression of the aptamer on ZAP's activity, the aptamer expressing plasmid was transfected into 
293TRex-ZAP or Rat2TRex-ZAP cells with pMLV-luc and pRL-TK. At $6 \mathrm{~h}$ posttransfection, the cells were mock-treated or treated with tetracycline to induce ZAP expression. At $48 \mathrm{~h}$ posttransfection, luciferase activities were measured. Fold inhibition by ZAP was calculated as the normalized luciferase activity in the mock-treated cells divided by the normalized luciferase activity in the tetracyclinetreated cells. Relative fold inhibition was calculated as the fold inhibition in the presence of the ZAP-binding aptamer divided by the fold inhibition in the presence of control RNA. The statistical significance of the data was analyzed with the SPSS program.

\section{ACKNOWLEDGEMENTS}

We thank Xudong Zhao of the core facility of the Institute of Biophysics for technical support. This work was supported in part by Grants (to G.G.) from National Natural Science Foundation of China (Grant Nos. 30470092 and 30530020) and National Basic Research Program of China (973 Program) (Grant No. 2006CB504302) of China.

\section{ABBREVIATIONS}

ARE, AU-rich element; EBOV, Ebola virus; HIV, Human immunodeficiency virus; MARV, Marburg virus; MLV, Moloney murine leukemia virus; SELEX, Systematic Evolution of Ligands by Exponential enrichment; SINV, Sindbis virus; TTP, tristetraproline; UTR, untranslated regions; ZAP, Zinc finger antiviral protein; ZRE, ZAP responsive element

\section{REFERENCES}

Barreau, C., Paillard, L., and Osborne, H.B. (2005). AU-rich elements and associated factors: are there unifying principles? Nucleic Acids Res 33, 7138-7150.

Bick, M.J., Carroll, J.W., Gao, G., Goff, S.P., Rice, C.M., and MacDonald, M.R. (2003). Expression of the zinc-finger antiviral protein inhibits alphavirus replication. J Virol 77, 11555-11562.

Blackshear, P.J. (2002). Tristetraprolin and other $\mathrm{CCCH}$ tandem zincfinger proteins in the regulation of mRNA turnover. Biochem Soc Trans 30, 945-952.

Carrick, D.M., Lai, W.S., and Blackshear, P.J. (2004). The tandem $\mathrm{CCCH}$ zinc finger protein tristetraprolin and its relevance to cytokine mRNA turnover and arthritis. Arthritis Res Ther 6, 248-264.

Chen, G., Guo, X., Lv, F., Xu, Y., and Gao, G. (2008). p72 DEAD box RNA helicase is required for optimal function of the zinc-finger antiviral protein. Proc Natl Acad Sci U S A 105, 4352-4357.

DuBois, R.N., McLane, M.W., Ryder, K., Lau, L.F., and Nathans, D. (1990). A growth factor-inducible nuclear protein with a novel cysteine/histidine repetitive sequence. J Biol Chem 265, 19185-19191.

Ellington, A.D., and Szostak, J.W. (1990). In vitro selection of RNA molecules that bind specific ligands. Nature 346, 818-822.

Gao, G., Guo, X., and Goff, S.P. (2002). Inhibition of retroviral RNA production by ZAP, a CCCH-type zinc finger protein. Science 297, 1703-1706.

Guo, X., Carroll, J.W., Macdonald, M.R., Goff, S.P., and Gao, G. (2004). The zinc finger antiviral protein directly binds to specific viral mRNAs through the $\mathrm{CCCH}$ zinc finger motifs. J Virol 78, 12781-12787.

Guo, X., Ma, J., Sun, J., and Gao, G. (2007). The zinc-finger antiviral protein recruits the RNA processing exosome to degrade the target mRNA. Proc Natl Acad Sci U S A 104, 151-156.

Jayasena, S.D. (1999). Aptamers: an emerging class of molecules that rival antibodies in diagnostics. Clin Chem 45, 1628-1650.

Lai, W.S., Carballo, E., Strum, J.R., Kennington, E.A., Phillips, R.S., and Blackshear, P.J. (1999). Evidence that tristetraprolin binds to AU-rich elements and promotes the deadenylation and destabilization of tumor necrosis factor alpha mRNA. Mol Cell Biol 19, $4311-4323$.

Lai, W.S., Carballo, E., Thorn, J.M., Kennington, E.A., and Blackshear, P.J. (2000). Interactions of $\mathrm{CCCH}$ zinc finger proteins with mRNA. Binding of tristetraprolin-related zinc finger proteins to Aurich elements and destabilization of mRNA. J Biol Chem 275, 17827-17837.

Müller, S., Möller, P., Bick, M.J., Wurr, S., Becker, S., Günther, S., and Kümmerer, B.M. (2007). Inhibition of filovirus replication by the zinc finger antiviral protein. J Virol 81, 2391-2400.

Tuerk, C., and Gold, L. (1990). Systematic evolution of ligands by exponential enrichment: RNA ligands to bacteriophage T4 DNA polymerase. Science 249, 505-510.

Tuerk, C., MacDougal, S., and Gold, L. (1992). RNA pseudoknots that inhibit human immunodeficiency virus type 1 reverse transcriptase. Proc Natl Acad Sci U S A 89, 6988-6992.

Yamamoto, R., Katahira, M., Nishikawa, S., Baba, T., Taira, K., and Kumar, P.K. (2000). A novel RNA motif that binds efficiently and specifically to the Ttat protein of HIV and inhibits the transactivation by Tat of transcription in vitro and in vivo. Genes Cells 5 , 371-388.

Zuker, M. (2003). Mfold web server for nucleic acid folding and hybridization prediction. Nucleic Acids Res 31, 3406-3415. 